\title{
The Kinase STK3 Interacts with the Viral Structural Protein VP1 and Inhibits Foot-and-Mouth Disease Virus Replication
}

\author{
Huisheng Liu, ${ }^{1}$ Qiao Xue, ${ }^{1}$ Qiaoying Zeng, ${ }^{1}$ Zixiang Zhu, ${ }^{2}$ and Haixue Zheng ${ }^{2}$ \\ ${ }^{1}$ Laboratory of Veterinary Microbiology, College of Veterinary Medicine, Gansu Agricultural University, Lanzhou, China \\ ${ }^{2}$ State Key Laboratory of Veterinary Etiological Biology, National Foot and Mouth Diseases Reference Laboratory, \\ Key Laboratory of Animal Virology of Ministry of Agriculture, Lanzhou Veterinary Research Institute, \\ Chinese Academy of Agricultural Sciences, Lanzhou 730046, China \\ Correspondence should be addressed to Qiaoying Zeng; zengqy@gsau.edu.cn and Haixue Zheng; haixuezheng@163.com
}

Received 28 June 2017; Accepted 14 September 2017; Published 31 October 2017

Academic Editor: Yi-Ping Liu

Copyright ( 2017 Huisheng Liu et al. This is an open access article distributed under the Creative Commons Attribution License, which permits unrestricted use, distribution, and reproduction in any medium, provided the original work is properly cited.

Foot-and-mouth disease virus (FMDV) is the etiological agent of FMD, which affects domestic and wild cloven-hoofed animals. The structural protein VP1 plays an important role in FMDV pathogenesis. However, the interacting partners of VP1 in host cells and the effects of these interactions in FMDV replication remain incompletely elucidated. Here, we identified a porcine cell protein, serine/threonine kinase 3 (STK3), which interacts with FMDV VP1 using the yeast two-hybrid system. The VP1-STK3 interaction was further confirmed by coimmunoprecipitation experiments in human embryonic kidney 293T and porcine kidney 15 (PK15) cells. The carboxyl-terminal region (amino acids 180-214) of VP1 was essential for its interaction with STK3. The effects of overexpression and underexpressing of STK3 in PK-15 cells were assessed, and the results indicated that STK3 significantly inhibited FMDV replication. Our data expand the role of STK3 during viral infection, provide new information regarding the host cell kinases that are involved in viral replication, and identify potential targets for future antiviral strategies.

\section{Background}

Foot-and-mouth disease virus (FMDV), a positive-sense, single-stranded RNA virus, is the etiological agent of FMD, which affects domestic and wild cloven-hoofed animals, including cattle, pigs, sheep, goats, camelids, and deer $[1,2]$. To date, seven serotypes (A, O, C, Asia, SAT1, SAT2, and SAT3) and numerous subtypes of FMDV have been identified, and no cross-protection has been reported among the different serotypes $[3,4]$. The FMDV genome is approximately $8.5 \mathrm{~kb}$ in length, and it encodes a single polyprotein that is posttranslationally processed into four structural proteins (VP1, VP2, $\mathrm{VP} 3$, and VP4) and eight nonstructural proteins $\left(\mathrm{L}^{\mathrm{pro}}, 2 \mathrm{~A}, 2 \mathrm{~B}\right.$, $2 \mathrm{C}, 3 \mathrm{~A}, 3 \mathrm{~B}, 3 \mathrm{C}^{\text {pro }}$, and $3 \mathrm{D}$ ) [5].

The contribution of each of these proteins to virulence during an infection of a natural host is not completely clear. To date, the proteins $\mathrm{VP} 0, \mathrm{VP} 1, \mathrm{VP} 3, \mathrm{~L}^{\text {pro }}, 2 \mathrm{~B}, 3 \mathrm{~A}$, and $3 \mathrm{C}^{\text {pro }}$ have been reported to play roles in inhibiting or evading the host innate immune system [5-13]. In addition, some host cell proteins that interact with the FMDV proteins VP1, $2 \mathrm{C}$, and $3 \mathrm{~A}$ were identified by the yeast two-hybrid system [11, 14-16]. VP1, an important viral protein that plays an essential role in FMDV pathogenesis, carries the major neutralizing antigenic sites, and the VP1 gene has been used widely in epidemiological investigations of FMDV, vaccine development, and the establishment of diagnostic methods $[17,18]$.

To better understand the role of FMDV VP1 in viral replication and virulence, we aimed to identify new host cell proteins that interact with VP1 using the yeast two-hybrid system. Here, we report that VP1 binds to serine/threonine kinase 3 (STK3), a member of the mammalian STE20-like (MST) kinase family. The MST kinase family, which is related to the Hippo kinase in Drosophila melanogaster, consists of five related proteins: STK3 (also known as MST2), STK4 
(also known as MST1), STK24 (also known as MST3), STK25 (also known as YSK1), and STK26 (also known as MST4) [19]. STK3 and STK4 are the key elements of the mammalian Hippo pathway, which influences cell proliferation, organ size, autophagy, apoptosis, and various stress responses [1922]. Recent reports indicated that STK3 and STK4 play important roles in bacterial infections $[20,23,24]$.

The VP1-STK3 interaction identified by the yeast twohybrid system was further confirmed by coimmunoprecipitation experiments and an indirect immunofluorescence assay (IFA). Additionally, the amino acid residues in FMDV VP1 that mediate the interaction with STK3 were identified. Furthermore, overexpression of STK3 decreased FMDV replication within infected cells, while a knockdown of STK3 expression facilitated FMDV replication. Taken together, our data demonstrate an important antiviral role of STK3 in FMDV replication, and they expand the role of STK3.

\section{Materials}

2.1. Cells, Viruses, and Infection. Porcine kidney 15 (PK-15) cells and human embryonic kidney 293T (HEK293T) cells were cultured in Dulbecco's modified Eagle medium (Gibco) supplemented with $10 \%$ heat-inactivated fetal bovine serum (Gibco) and maintained at $37^{\circ} \mathrm{C}\left(5 \% \mathrm{CO}_{2}\right)$. The FMDV type $\mathrm{O}$ strain $\mathrm{O} / \mathrm{BY} / \mathrm{CHA} / 2010$ was used for viral infections as described previously [25].

2.2. Plasmids and Antibodies. The cDNA of porcine STK3 was amplified from PK-15 cells and cloned into the pcDNATM3.1/ myc-His(-)A vector (Invitrogen, Carlsbad, CA, USA) to yield the Myc-tagged expression construct (Myc-STK3). A FLAG-tagged VP1 construct and a series of FLAG-tagged truncated VP1 constructs were prepared in our laboratory. All constructed plasmids were analyzed and verified by DNA sequencing. The commercial antibodies used in this study include an anti-Myc monoclonal antibody (Santa Cruz Biotechnology, Dallas, TX, USA), an anti-FLAG monoclonal antibody (Santa Cruz Biotechnology), an anti-FLAG polyclonal antibody (Sigma-Aldrich, St. Louis, MO, USA), an anti-STK3 polyclonal antibody (Abcam, Cambridge, MA, USA), and an anti- $\beta$-actin monoclonal antibody (Santa Cruz Biotechnology). An anti-VP1 polyclonal antibody was prepared in our laboratory [6].

2.3. Yeast Two-Hybrid Screen. A cDNA expression library was constructed using PK-15 cells. Total RNA was extracted using the RNeasy Extraction Kit (Qiagen, Valen-cia, CA, USA). Contaminating genomic DNA was removed by DNase treatment using Turbo DNA-free DNase (Ambion, Austin, TX, USA). Subsequently, RNA quality was assessed using RNA nanochips on a Bioanalyzer 2100 (Agilent Technologies, Santa Clara, CA, USA). GAL4-activation domain-cellular protein fusions were used as prey. The FMDV strain type $\mathrm{O}$ strain O/BY/CHA/2010 VP1, which was expressed as an amino-terminal fusion to the GAL4-DNA-binding domain, was used as the bait protein. Histidine and adenine were used for reporter genes for growth selection. The porcine library contained more than $3 \times 10^{6}$ independent cDNA clones. For screening, a yeast strain expressing the VP1 protein was transformed with library plasmid DNA and selected on plates lacking tryptophan, leucine, histidine, and adenine. Once identified, positive library plasmids were recovered in Escherichia coli (E. coli) and sequenced to identify interacting cellular proteins. STK3 that was recovered from the library matched porcine STK3 (National Center for Biotechnology Information [NCBI] reference sequence GACC01000309.1).

2.4. Coimmunoprecipitation and Western Blot Analysis. HEK293T or PK-15 cells were seeded in $10-\mathrm{cm}$ dishes, and monolayer cells were cotransfected with various plasmids. Then, the cells were collected and lysed, and proteins were immunoprecipitated as described previously [7]. The target proteins were resolved by sodium dodecyl sulfatepolyacrylamide gel electrophoresis and transferred onto an Immobilon-P membrane (EMD Millipore, Billerica, MA, USA). The membrane was blocked and then incubated with primary and secondary antibodies as described previously [26]. Antibody-antigen complexes were visualized by enhanced chemiluminescence detection reagents (Thermo Fisher Scientific, Waltham, MA, USA).

2.5. Indirect Immunofluorescence Microscopy. HEK293T cells were grown on $\mathrm{Nunc}^{\mathrm{TM}}$ glass bottom dishes and transfected with various plasmids using Lipofectamine 3000 (Invitrogen) according to the manufacturer's protocol. At $24 \mathrm{~h}$ after transfection (hpt), the cells were treated as described previously [6].

2.6. Knockdown of STK3 Using a Small Interfering RNA (siRNA). The siRNAs used in this experiment were chemically synthesized by GenePharma (Beijing, China). The knockdown of endogenous STK3 in PK-15 cells was conducted by transfection with an STK3 siRNA. A nontargeting RNA (NC siRNA) was used as a negative control. siRNA transfection was performed using Lipofectamine 2000 (Invitrogen) according to the manufacturer's protocol. The target sequence for porcine STK3 was as follows:

\section{F: $5^{\prime}$-GCUGGAAAUAUUCUCCUUATT-3', R: 5'-UAAGGAGAAUAUUUCCAGCTT-3'.}

2.7. RNA Extraction and Quantitative Polymerase Chain Reaction ( $q P C R)$. Total RNAs were extracted using TRIzol ${ }^{\circledR}$ reagent (Invitrogen). The isolated RNA was reverse transcribed to cDNA using the Moloney murine leukemia virus reverse transcriptase (Promega, Madison, WI, USA) and random hexamer primers (TaKaRa, Shiga, Japan). The transcriptional level of the mRNA was quantified by qPCR using SYBR Premix Ex Taq reagents (TaKaRa) in the Mx3005P qPCR System (Agilent Technologies). The glyceraldehyde3-phosphate dehydrogenase (GAPDH) gene was used as an internal control. The qPCR primers used in this study are listed in Table 1. Relative fold changes of mRNA were calculated using the comparative cycle threshold (CT) $\left(2^{-\Delta \Delta C T}\right)$ 
TABLE 1: qPCR primers used in this study.

\begin{tabular}{lcc}
\hline Primers & Sequences $\left(5^{\prime}-3^{\prime}\right)$ & Use \\
\hline STK3-F & TTTTGGATGGCTCCTGAGGTAAT & Detection of porcine STK3 gene \\
STK3-R & TGAATGTTGGTGGTGGGTTTGTG & Detection of FMDV gene \\
FMDV-F & CACTGGTGACAGGCTAAGG & Detection of porcine GAPDH gene \\
FMDV-R & CCCTTCTCAGATTCCGAGT \\
GAPDH-F & ACATGGCCTCCAAGGAGTAAGA & \\
GAPDH-R & GATCGAGTTGGGGCTGTGACT & \\
\hline
\end{tabular}

method [27]. All the experiments were repeated three times with similar results. The data represent results from one of the triplicate experiments.

2.8. Statistical Analysis. Statistical analysis was performed using SPSS Statistics for Windows, Version 17.0 (SPSS Inc., Chicago, IL, USA). Student's $t$-test was used for a comparison of three independent experiments. A $p$ value $<0.05$ was considered statistically significant $(*)$; A $p$ value $<0.01$ was considered statistically highly significant $(* *)$.

\section{Results}

3.1. The FMDV Structural Protein VP1 Interacts with the Porcine Host Protein STK3. To date, the multiple functions of FMDV VP1 during viral infection remain unclear. To identify the host cellular proteins interacting with VP1 of FMDV, the FMDV VP1 was expressed as an amino-terminal fusion to the GAL4-DNA-binding domain. In the screen of a cDNA library of PK-15 cells using the yeast two-hybrid system, the plasmids in yeast were isolated, amplified in E. coli $\mathrm{DH} 5 \alpha$, and sequenced. Among them, one of these host proteins, identified as porcine STK3 (NCBI reference sequence GACC01000309.1), was selected for further immunoprecipitation analysis.

To confirm the interaction of VP1 and STK3, HEK$293 \mathrm{~T}$ cells were cotransfected with a Myc-STK3 expressing plasmid and a FLAG-VP1 expressing plasmid or an empty FLAG vector. Cell lysates were immunoprecipitated with an anti-Myc antibody and analyzed by western blotting. As shown in Figure 1(a), Myc-STK3 pulled down FLAGVP1, indicating an interaction between STK3 and VP1. The immunoprecipitated Myc-STK3 protein and the wholecell lysates were visualized by enhanced chemiluminescence detection reagents. Then, a reverse immunoprecipitation experiment was performed using an anti-FLAG antibody. It was observed that FLAG-VP1 also immunoprecipitated MycSTK3 (Figure 1(b)).

To further confirm that the FMDV VP1 and STK3 interaction occurs in vivo, $\mathrm{PK}-15$ cells were transfected with a FLAG-VP1 expressing plasmid or an empty FLAG vector. Cell lysates were immunoprecipitated with an anti-STK3 antibody and analyzed by western blotting. As shown in Figure 1(c),
STK3 pulled down FLAG-VP1. Then, a reverse immunoprecipitation experiment was performed using an anti-FLAG antibody. Similarly, the results showed that FLAG-VP1 also immunoprecipitated STK3 (Figure 1(d)).

An IFA was performed subsequently, and the results indicated an interaction between VP1 and STK3 (Figure 1(e)). Taken together, these results confirm that FMDV VP1 interacts with STK3.

3.2. The Carboxyl-Terminal Region (Amino Acids 180-214) of VP1 Is Essential for the VP1-STK3 Interaction. To identify the structural domains of FMDV VP1 that are responsible for the STK3 interaction, a series of truncated mutants of FMDV VP1 was generated (Figure 2(a)). HEK-293T cells were cotransfected with a Myc-STK3 expressing plasmid and a FLAG-VP1 expressing plasmid, FLAG-VP1 mutants expressing plasmids or an empty FLAG vector. Cell lysates were immunoprecipitated with an anti-FLAG antibody and analyzed by western blotting. The results suggested that the carboxyl-terminal region (amino acids 115-214) of VP1 interacts with STK3 (Figure 2(b)). Next, we examined which amino acids in carboxyl-terminal region of VP1 are required for its interaction with STK3. The results indicated that amino acids 180-214 of FMDV VP1 are essential for the STK3 interaction (Figure 2(c)).

3.3. FMDV Infection Decreases Endogenous STK3 Protein Abundance. The abundance of endogenous STK3 during an FMDV infection is unclear. Therefore, PK-15 cells were infected with equal amounts of FMDV at a multiplicity of infection (MOI) of 0.5. The STK3 mRNA level and the STK3 protein abundance were determined and compared over time. The results indicated that both the STK3 mRNA level and STK3 protein abundance decreased at an early infection time (Figure 3). Taken together, we speculate that FMDV reduces the expression of STK3 protein by inhibiting its mRNA level, not at translational level.

3.4. STK3 Inhibits FMDV Replication during Virus Infection. To assess the role of STK3 in FMDV replication, we evaluated the FMDV yield in PK-15 cells that were transfected with different doses of a Myc-STK3 expressing plasmid. At $24 \mathrm{hpt}$, the cells were infected with equal amounts of FMDV (MOI 


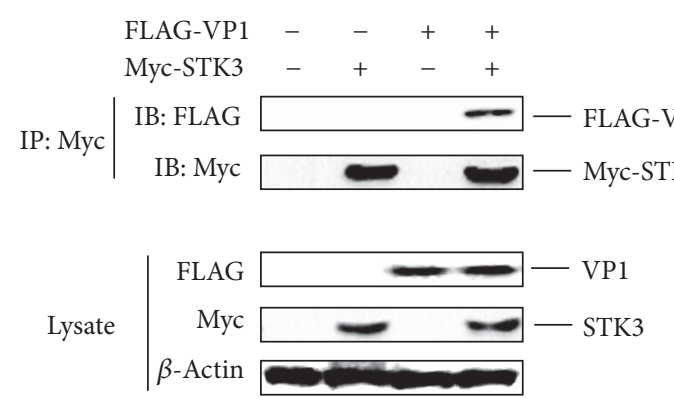

(a)

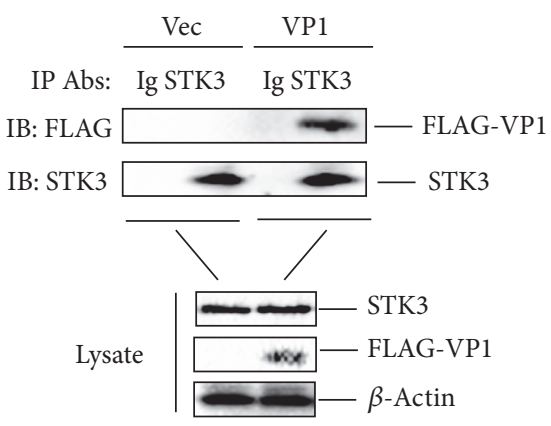

(c)
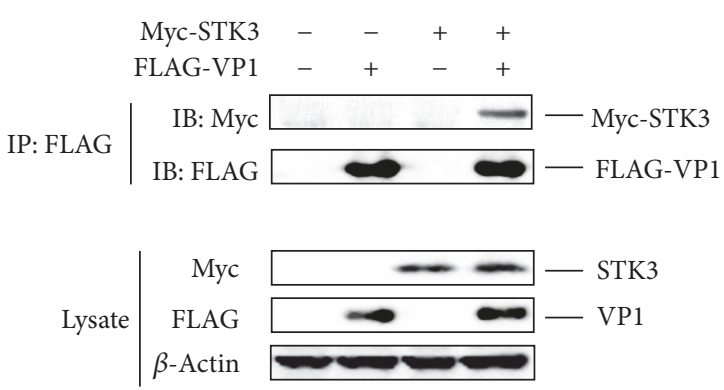

(b)

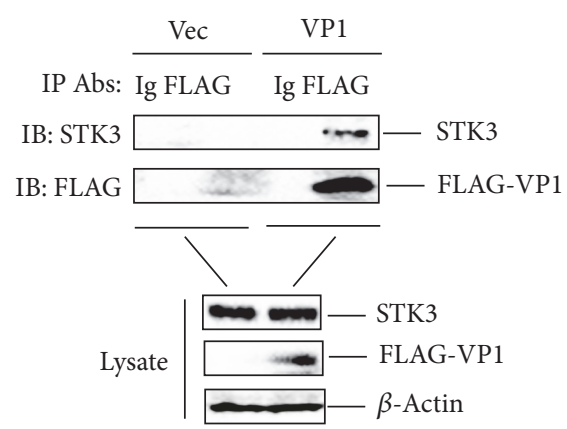

(d)

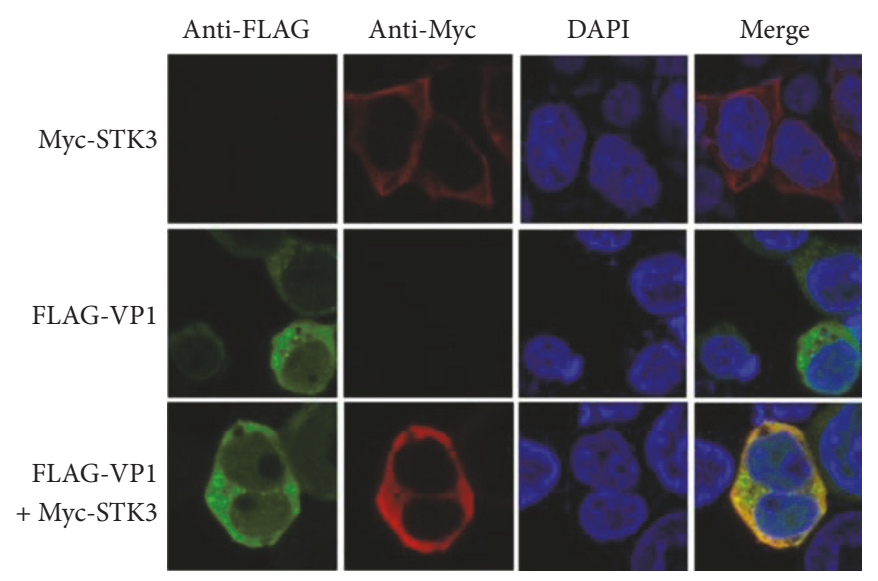

(e)

FIGURE 1: STK3 interacts with VP1. (a) HEK-293T cells were seeded in 10-cm dish, and the monolayer cells were transfected with $8 \mu \mathrm{g}$ of a Myc- STK3 expressing plasmid (+), $8 \mu \mathrm{g}$ of a FLAG-VP1 expressing plasmid (+), $8 \mu \mathrm{g}$ of an empty FLAG vector (-), or $8 \mu \mathrm{g}$ of an empty Myc vector (-). At $24 \mathrm{hpt}$, the cells were lysed, and the lysates were immunoprecipitated with mouse anti-Myc or mouse normal IgG antibodies and subjected to western blotting. The immunoprecipitated antibody-antigen complexes and whole-cell lysates were analyzed by immunoblotting using anti-FLAG, anti-Myc, or anti- $\beta$-actin antibodies. (b) Similar transfection and immunoprecipitation experiments were performed as described above. However, the lysates were immunoprecipitated with mouse anti-FLAG or mouse normal IgG antibodies and subjected to western blotting. (c) PK-15 cells were seeded in $10 \mathrm{~cm}$ dish, and the monolayer cells were transfected with $10 \mu \mathrm{g}$ of a FLAG-VP1 expressing plasmid or $10 \mu \mathrm{g}$ of an empty FLAG vector. The cells were lysed at $30 \mathrm{hpt}$. Cell lysates were immunoprecipitated with goat anti-STK3 and goat IgG antibodies and subjected to western blotting. The whole-cell lysates and immunoprecipitated antibody-antigen complexes were analyzed by immunoblotting using anti-STK3, anti-FLAG, or anti- $\beta$-actin antibodies. (d) Similar infection and immunoprecipitation experiments were performed as described above. However, the lysates were immunoprecipitated with mouse anti-FLAG or mouse normal IgG antibodies and subjected to western blotting. (e) HEK293T cells were seeded in Nunc glass bottom dishes, and the monolayer cells were transfected with $1.5 \mu \mathrm{g}$ of a Myc-STK3 expressing plasmid, $1.5 \mu \mathrm{g}$ of a FLAG-VP1 expressing plasmid, or $1.5 \mu \mathrm{g}$ of an empty vector. At $24 \mathrm{hpt}$, the expression of Myc-STK3 and FLAG-VP1 was detected by an IFA analysis. Cells were double-immunostained for Myc-STK3 (red) and FLAG-VP1 (green); cellular nuclei were counterstained with $4^{\prime}$,6-diamidino-2-phenylindole (DAPI) (blue). 


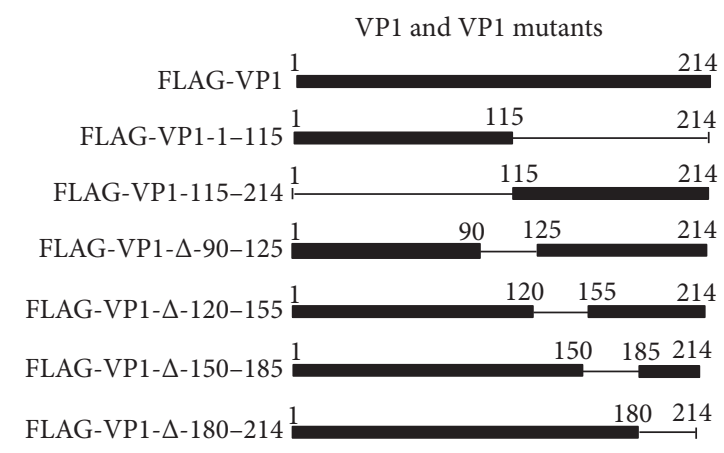

(a)

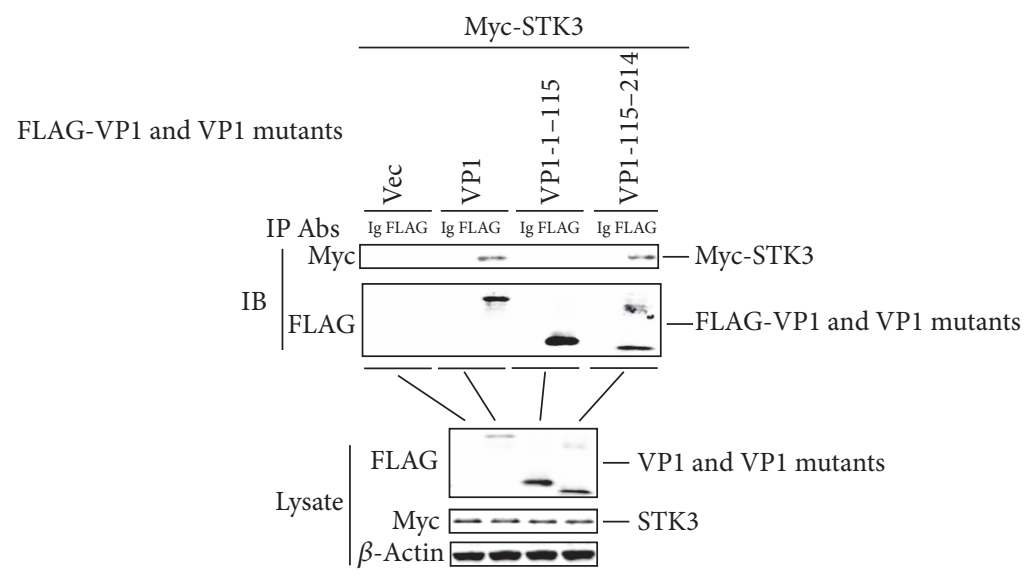

(b)

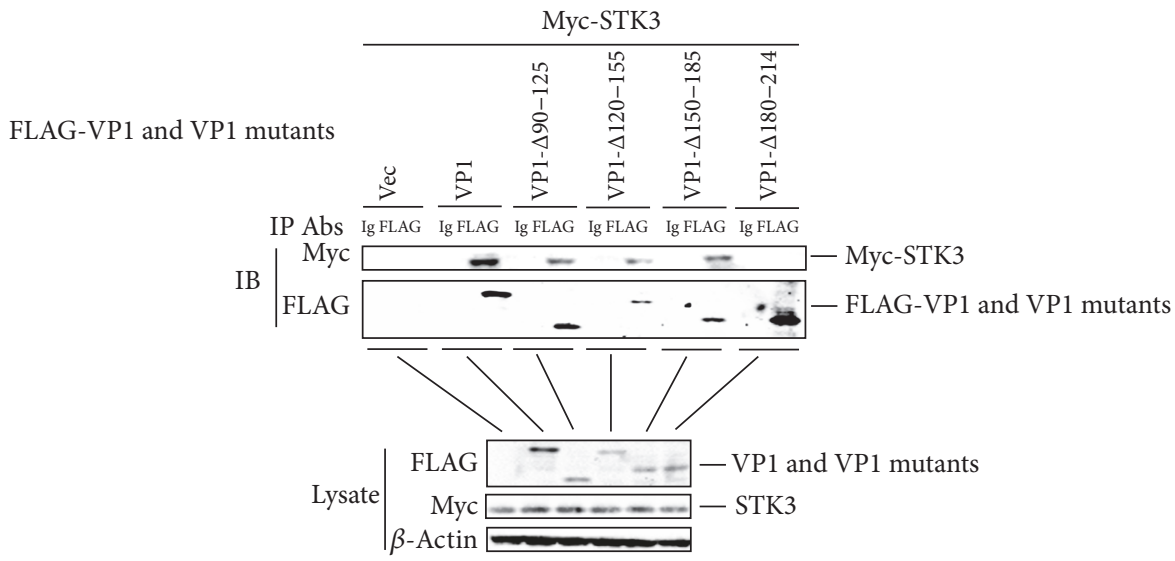

(c)

FIGURE 2: STK3 interacts with VP1 truncated mutants. (a) Schematics showing a series of FLAG-tagged truncated VP1 mutants. (b) HEK-293T cells were seeded in a 10-cm dish, and the monolayer cells were transfected with $8 \mu \mathrm{g}$ of a Myc-STK3 expressing plasmid, $8 \mu \mathrm{g}$ of a FLAG-VP1 expressing plasmid, $8 \mu \mathrm{g}$ of FLAG-VP1 mutants expressing plasmids, or $8 \mu \mathrm{g}$ of an empty FLAG vector. The cells were lysed at $24 \mathrm{hpt}$. The lysates were immunoprecipitated with mouse anti-FLAG or mouse IgG antibodies and subjected to western blotting. The immunoprecipitated antibody-antigen complexes and whole-cell lysates were analyzed by anti-FLAG, anti-Myc, or anti- $\beta$-actin antibodies. (c) Similar transfection and immunoprecipitation experiments were performed as described above.

$0.5)$ for $12 \mathrm{~h}$. The FMDV RNA level and protein abundance were compared. As shown in Figure 4(a), overexpression of STK3 significantly suppressed FMDV RNA levels and protein abundance in a dose-dependent manner.
The replication level of FMDV in cells in which STK3 expression was downregulated was further assessed. PK-15 cells were transfected with STK3 siRNA or NC siRNA for $48 \mathrm{~h}$, and then they were infected with equal amounts of 

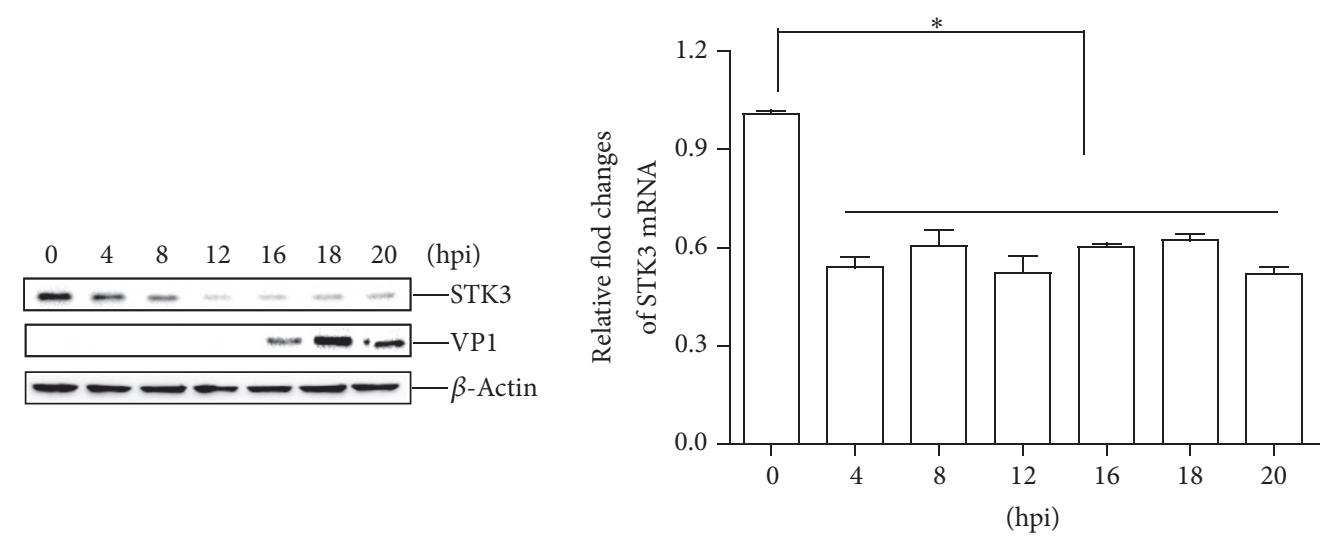

FIGURE 3: FMDV infection decreases the abundance of the endogenous STK3 protein. PK-15 cells were seeded in $3.5 \mathrm{~cm}$ dish, and the monolayer cells were infected with FMDV (MOI 0.5). The cells were collected at the indicated time points $(0,4,8,12,16,18$, and $20 \mathrm{~h})$. The expression of STK3 mRNA was determined by qPCR assay (b). The expression of endogenous STK3 and viral VP1 proteins was determined by western blotting (a).

FMDV (MOI 0.5). The viral RNA level and the abundance of the VP1 and STK3 proteins in the STK3 siRNA-transfected and NC siRNA-transfected cells were compared at 0,6 , and $12 \mathrm{~h}$ after FMDV infection. The results indicated that the FMDV replication level increased significantly in STK3 siRNA-transfected cells (Figure 4(b)).

Taken together, these results demonstrate the important antiviral role of STK3 in FMDV replication.

\section{Discussion}

Viral virulence is dependent on a balanced interaction between viral and cellular proteins. Studies have shown that host protein kinases are important regulators of virushost interaction and that they play important roles in viral replication [28]. The potential mechanisms by which FMDV proteins interact with host cell proteins are not fully understood. Here, for the first time, we determined that the FMDV structural protein VP1 interacts with porcine STK3 using the yeast two-hybrid system. In concordance with this result, we also demonstrated the interaction of VP1 with STK3 in HEK293T and PK-15 cells using coimmunoprecipitation experiments. Our data also showed that the carboxyl-terminal region (180-214) of FMDV VP1 is essential for the interaction with STK3. The G-H loop, containing amino acids $134-160$ of VP1, plays a significant role in VP1 functions [17]. However, our results indicate that the G-H loop region is not responsible for the interaction of VP1 with STK3.

In humans, studies have indicated that patients lacking STK4, which is a closely related paralog of STK3, showed $\mathrm{T}$ - and B-cell lymphopenia and recurrent bacterial and viral infections $[29,30]$. STK4, but not STK3, can shut off cytosolic antiviral defense through IRF3 phosphorylation [31]. Besides, downregulation of STK3 expression decreased influenza replication, which indicates that STK3 can facilitate viral replication [32]. In veterinary research, the roles of STK3 in bacterial or viral infections have not been reported. Taken together, the antiviral roles and the exact mechanisms of action of STK3 have not been clarified.

In this study, overexpression and underexpression of STK3 in PK-15 cells were performed. The results confirmed the antiviral role of STK3 in FMDV replication. A recent study showed that Hippo pathway is a potent regulator of cellular antiviral response [33]. STK3 is involved in the Hippo pathway that activates various functions [19]. Therefore, we speculate that STK3 inhibits FMDV replication via the Hippo pathway. Determining the specific antiviral mechanisms mediated by the Hippo pathway requires further studies.

In conclusion, our results showed, for the first time, that the porcine cellular protein STK3 interacts with FMDV VP1. The VP1-STK3 interaction may be critical for modulating viral replication. The results also described a novel antiviral role of STK3 during FMDV infection. This presents possible new VP1 functions in viral pathogenesis, and further studies need to be performed to identify other cellular proteins that may interact with FMDV proteins, which will promote a better understanding of FMDV pathogenesis.

\section{Conflicts of Interest}

The authors declare that they have no conflicts of interest.

\section{Acknowledgments}

The authors acknowledge the Facility Center Department, Lanzhou Veterinary Research Institute, for helpful support during the course of the work. This study was supported by 

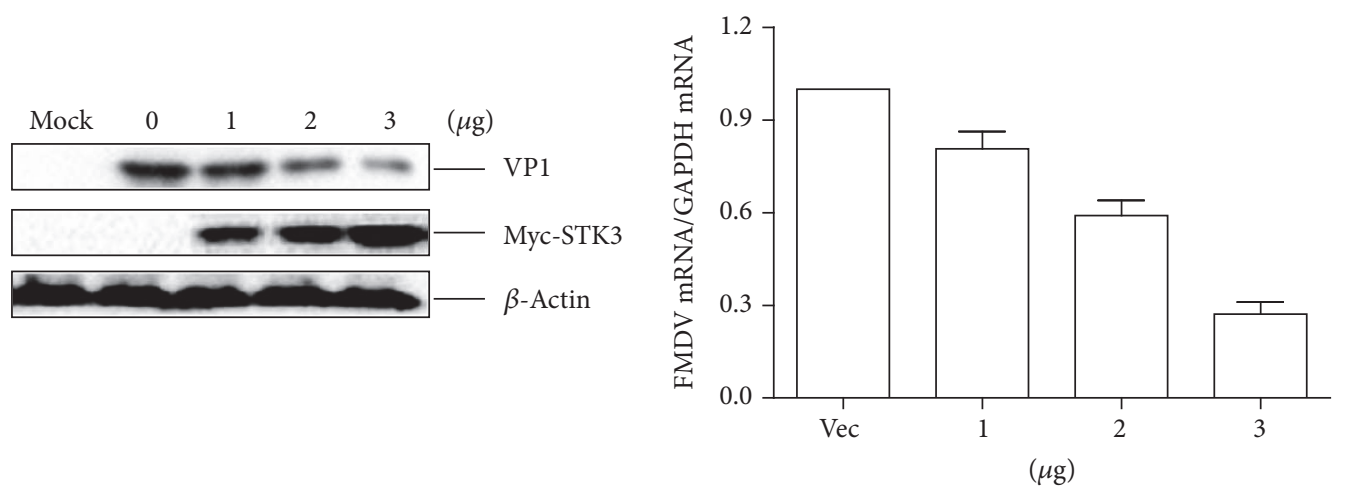

(a)
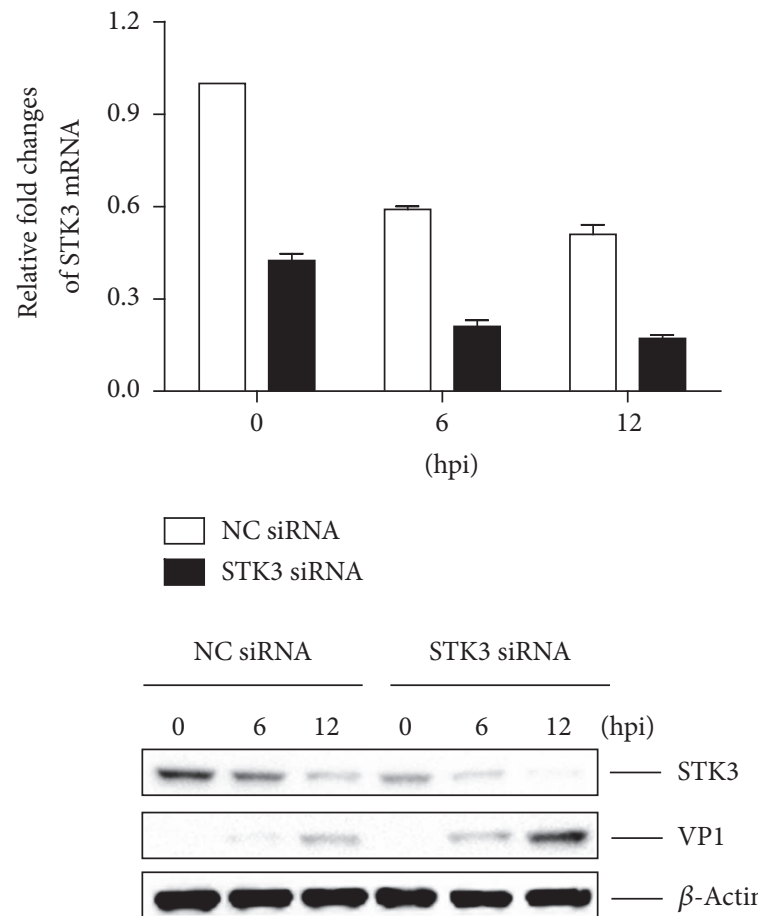

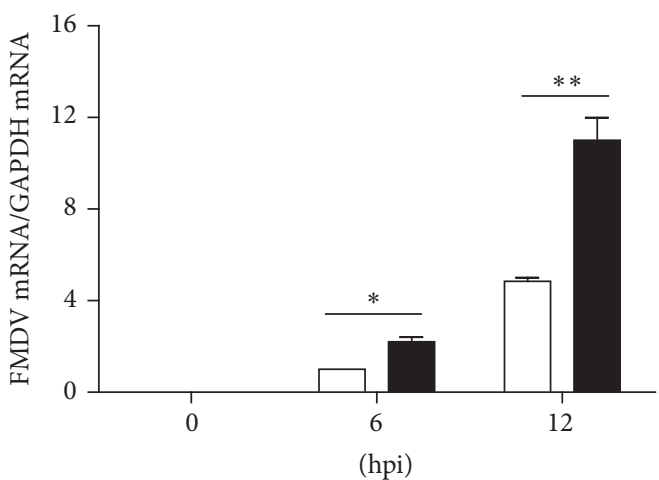

NC SiRNA

STK3 siRNA

(b)

FIGURE 4: STK3 inhibits FMDV replication during virus infection. (a) PK-15 cells were seeded in 6-well plates, and the monolayer cells were transfected with 1,2, and $3 \mu \mathrm{g}$ of a Myc-STK3 expressing plasmid and $3 \mu \mathrm{g}$ of an empty Myc vector. The empty Myc vector was used in the transfection process to ensure that the same amount of cells received the same amount of total plasmids. At $24 \mathrm{hpt}$, the cells were mock infected or infected with FMDV (MOI 0.5) for $12 \mathrm{~h}$. The expression of viral RNA and the VP1 protein was detected by qPCR assay and western blotting, respectively. (b) PK-15 cells were seeded in $3.5 \mathrm{~cm}$ dish, and the monolayer cells were transfected with $150 \mathrm{nM}$ of STK3 siRNA or NC siRNA for $48 \mathrm{~h}$, followed by infection with equal amounts of FMDV (MOI 0.5). The cells were collected at the indicated time points $(0,6$, and $12 \mathrm{~h}$ ). Expression of STK3 mRNA and viral RNA was determined by qPCR assay. Expression of STK3 and the viral VP1 protein was detected by western blotting.

grants from National Natural Science Foundation of China (nos. 31302106 and 31260616).

\section{References}

[1] M. J. Grubman and B. Baxt, "Foot-and-mouth disease," Clinical Microbiology Reviews, vol. 17, no. 2, pp. 465-493, 2004.

[2] G. J. Belsham, "Distinctive features of foot-and-mouth disease virus, a member of the picornavirus family; aspects of virus protein synthesis, protein processing and structure," Progress in Biophysics and Molecular Biology, vol. 60, no. 3, pp. 241-260, 1993.
[3] Y.-M. Dong, J.-C. Cai, H.-T. Chen, and L. Chen, "Protection of a novel epitope-RNA VLP double-effective VLP vaccine for foot-and-mouth disease," Antiviral Research, vol. 134, pp. 108116, 2016.

[4] C. Carrillo, E. R. Tulman, G. Delhon et al., "Comparative genomics of foot-and-mouth disease virus," Journal of Virology, vol. 79, no. 10, pp. 6487-6504, 2005.

[5] D. Li, C. Lei, Z. Xu et al., "Foot-and-mouth disease virus nonstructural protein $3 \mathrm{~A}$ inhibits the interferon- $\beta$ signaling pathway," Scientific Reports, vol. 6, Article ID 21888, 2016.

[6] Z. Zhu, G. Wang, F. Yang et al., "Foot-and-mouth disease virus viroporin $2 \mathrm{~B}$ antagonizes RIG-I-mediated antiviral effects by 
inhibition of its protein expression," Journal of Virology, vol. 90, no. 24, pp. 11106-11121, 2016.

[7] D. Li, W. Yang, F. Yang et al., "The VP3 structural protein of footand-mouth disease virus inhibits the IFN- $\beta$ signaling pathway," The FASEB Journal, vol. 30, no. 5, pp. 1757-1766, 2016.

[8] D. Li, J. Wei, F. Yang et al., "Foot-and-mouth disease virus structural protein VP3 degrades Janus kinase 1 to inhibit IFN- $\gamma$ signal transduction pathways," Cell Cycle, vol. 15, no. 6, pp. 850860, 2016

[9] Y. Liu, Z. Zhu, M. Zhang, and H. Zheng, "Multifunctional roles of leader protein of foot-and-mouth disease viruses in suppressing host antiviral responses," Veterinary Research, vol. 46, no. 1, article 127, 2015.

[10] Y. Du, J. Bi, J. Liu et al., "3Cpro of foot-and-mouth disease virus antagonizes the interferon signaling pathway by blocking STAT1/STAT2 nuclear translocation," Journal of Virology, vol. 88, no. 9, pp. 4908-4920, 2014.

[11] X. Li, J. Wang, J. Liu et al., "Engagement of soluble resistancerelated calcium binding protein (sorcin) with foot-and-mouth disease virus (FMDV) VP1 inhibits type I interferon response in cells," Veterinary Microbiology, vol. 166, no. 1-2, pp. 35-46, 2013.

[12] D. Wang, L. Fang, K. Li et al., "Foot-and-mouth disease virus 3C protease cleaves NEMO to impair innate immune signaling," Journal of Virology, vol. 86, no. 17, pp. 9311-9322, 2012.

[13] H. F. Mu, X. S. Ma, Z. K. Luo, J. Zhang, and H. X. Zheng, "Structural protein VP0 of foot-and-mouth disease virus inhibits type I interferon signaling pathway," Acta Microbiologica Sinica, pp. 1-11, 2017.

[14] D. P. Gladue, V. O’Donnell, R. Baker-Bransetter et al., "Interaction of foot-and-mouth disease virus nonstructural protein $3 \mathrm{~A}$ with host protein DCTN3 is important for viral virulence in cattle," Journal of Virology, vol. 88, no. 5, pp. 2737-2747, 2014.

[15] D. P. Gladue, V. O’Donnell, R. Baker-Branstetter et al., "Footand-Mouth disease virus modulates cellular vimentin for virus survival," Journal of Virology, vol. 87, no. 12, pp. 6794-6803, 2013.

[16] D. P. Gladue, V. O’Donnell, R. Baker-Branstetter et al., "Footand-mouth disease virus nonstructural protein 2C interacts with beclinl, modulating virus replication," Journal of Virology, vol. 86, no. 22, pp. 12080-12090, 2012.

[17] K. Lian, F. Yang, Z. Zhu et al., “The VP1 S154D mutation of type Asial foot-and-mouth disease virus enhances viral replication and pathogenicity," Infection, Genetics and Evolution, vol. 39, pp. 113-119, 2016.

[18] Q. Zhang, X. Liu, Y. Fang et al., "Evolutionary analysis of structural protein gene VP1 of foot-and-mouth disease virus serotype asia 1," The Scientific World Journal, vol. 2015, Article ID 734253, 2015.

[19] B. J. Thompson and E. Sahai, "MST kinases in development and disease," The Journal of Cell Biology, vol. 210, no. 6, pp. 871-882, 2015.

[20] D. S. Wilkinson, J. S. Jariwala, E. Anderson et al., "Phosphorylation of LC3 by the hippo kinases STK3/STK4 is essential for autophagy," Molecular Cell, vol. 57, no. 1, pp. 55-68, 2015.

[21] S. Xu, H. Wu, H. Nie et al., "AIF downregulation and its interaction with STK3 in renal cell carcinoma," PLoS ONE, vol. 9, no. 7, Article ID e100824, 2014.

[22] K.-K. Lee, T. Ohyama, N. Yajima, S. Tsubuki, and S. Yonehara, "MST, a physiological caspase substrate, highly sensitizes apoptosis both upstream and downstream of caspase activation," The Journal of Biological Chemistry, vol. 276, no. 22, pp. 19276-19285, 2001.
[23] L. M. Stuart and A. Lacy-Hulbert, "De-Mst-ifying microbicidal killing," Nature Immunology, vol. 16, no. 11, pp. 1107-1108, 2015.

[24] J. Geng, X. Sun, P. Wang et al., "Kinases Mst1 and Mst2 positively regulate phagocytic induction of reactive oxygen species and bactericidal activity," Nature Immunology, vol. 16, no. 11, pp. 1142-1152, 2015.

[25] H. Zheng, J. Guo, Y. Jin et al., "Engineering foot-and-mouth disease viruses with improved growth properties for vaccine development," PLoS ONE, vol. 8, no. 1, Article ID e55228, 2013.

[26] Z. Zhu, Z. Shi, W. Yan et al., "Nonstructural Protein 1 of Influenza A Virus Interacts with Human Guanylate-Binding Protein 1 to Antagonize Antiviral Activity," PLoS ONE, vol. 8, no. 2, Article ID e55920, 2013.

[27] T. D. Schmittgen and K. J. Livak, "Analyzing real-time PCR data by the comparative CT method," Nature Protocols, vol. 3, no. 6, pp. 1101-1108, 2008.

[28] C. Hutterer, S. K. Wandinger, S. Wagner et al., "Profiling of the kinome of cytomegalovirus-infected cells reveals the functional importance of host kinases Aurora A, ABL and AMPK," Antiviral Research, vol. 99, no. 2, pp. 139-148, 2013.

[29] N. T. Nehme, J. P. Schmid, F. Debeurme et al., "MST1 mutations in autosomal recessive primary immunodeficiency characterized by defective naive T-cell survival," Blood, vol. 119, no. 15, pp. 3458-3468, 2012.

[30] H. Abdollahpour, G. Appaswamy, D. Kotlarz et al., "The phenotype of human STK4 deficiency," Blood, vol. 119, no. 15, pp. 3450-3457, 2012.

[31] F. Meng, R. Zhou, S. Wu et al., "Mst1 shuts off cytosolic antiviral defense through IRF3 phosphorylation," Genes \& Development, vol. 30, no. 9, pp. 1086-1100, 2016.

[32] C. Atkins, C. W. Evans, B. Nordin et al., "Global human-kinase screening identifies therapeutic host targets against influenza," Journal of Biomolecular Screening, vol. 19, no. 6, pp. 936-946, 2014.

[33] Q. Zhang, F. Meng, S. Chen et al., "Hippo signalling governs cytosolic nucleic acid sensing through YAP/TAZ-mediated TBK1 blockade," Nature Cell Biology, vol. 19, no. 4, pp. 362-374, 2017. 

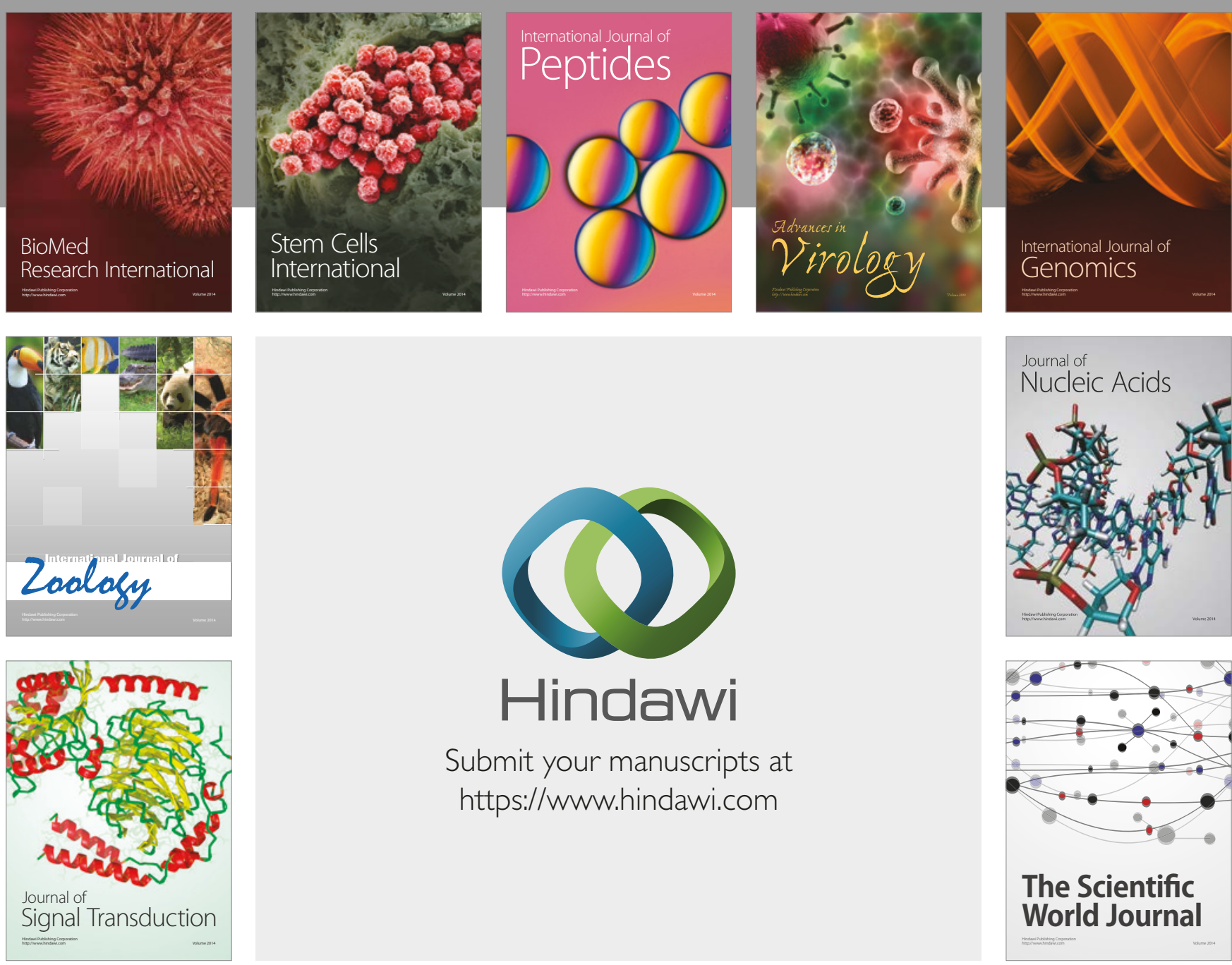

Submit your manuscripts at

https://www.hindawi.com
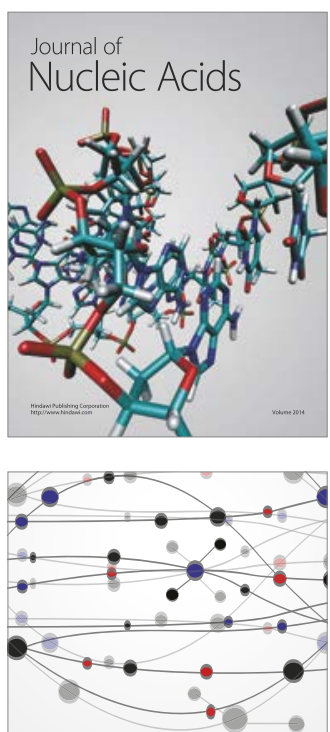

The Scientific World Journal

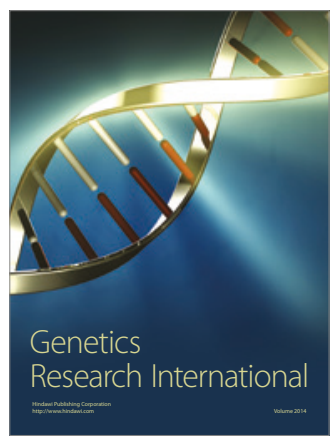

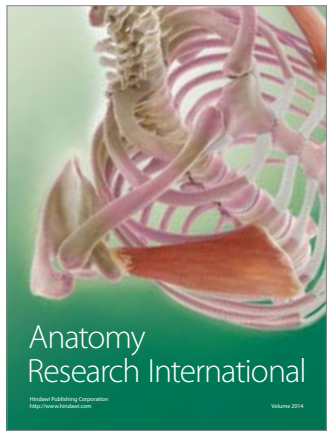

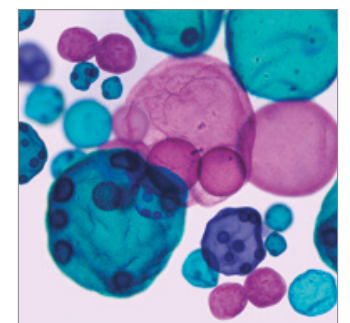

International Journal of Microbiology
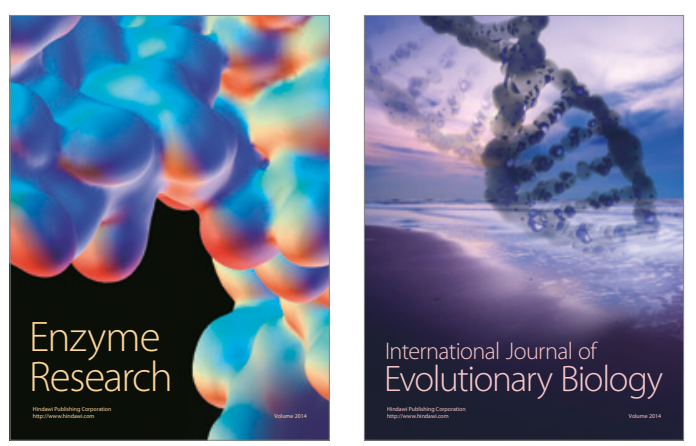
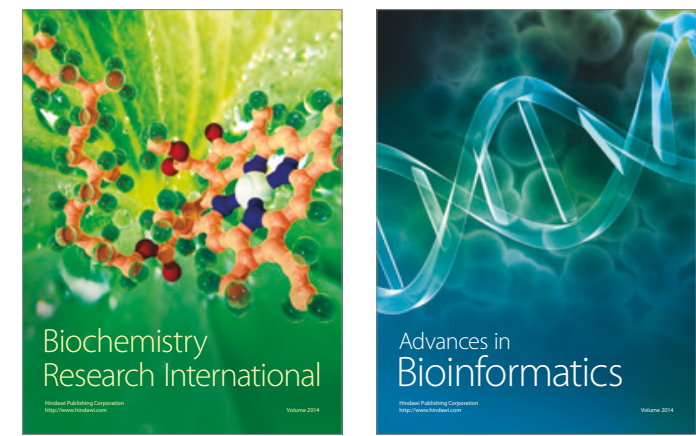

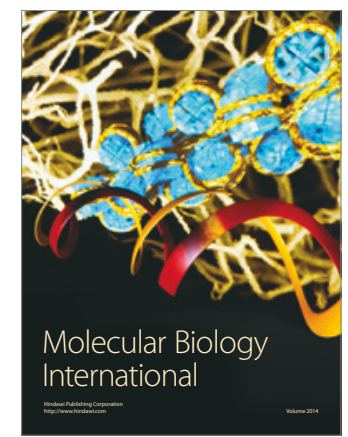

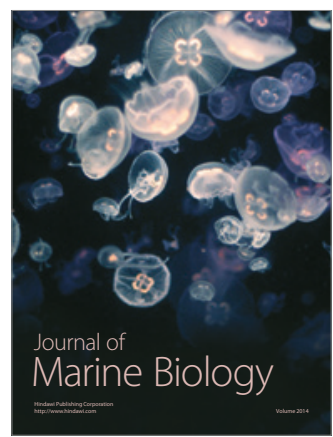

